\title{
The role of TRPC6 in $\alpha 1-A R$ activation-induced calcium signal changes in human podocytes
}

\author{
Derun Wang ${ }^{1,2}$, Qin Wang ${ }^{3}$, Tianrong Ji ${ }^{3}$, He Yang ${ }^{3}$, Fanwu Kong ${ }^{3}$, Jundong Jiao ${ }^{3,4}$ \\ ${ }^{1}$ Department of Cadre Ward, ${ }^{2}$ Department of General Practice, The First Affiliated Hospital of Harbin Medical University, Harbin, China; \\ ${ }^{3}$ Department of Nephrology, The Second Affiliated Hospital of Harbin Medical University, Harbin, China; ${ }^{4}$ Institute of Nephrology, Harbin Medical \\ University, Harbin, China \\ Contributions: (I) Conception and design: D Wang, J Jiao; (II) Administrative support: J Jiao, Q Wang; (III) Provision of study materials: T Ji, He \\ Yang, F Kong, D Wang; (IV) Collection and assembly of data: D Wang, J Jiao; (V) Data analysis and interpretation: T Ji, H Yang, F Kong, D Wang; (VI) \\ Manuscript writing: All authors; (VII) Final approval of manuscript: All authors. \\ Correspondence to: Jundong Jiao. Department of Nephrology, The Second Affiliated Hospital of Harbin Medical University, Harbin, China. \\ Email: jiaojundong@163.com.
}

\begin{abstract}
Background: An accumulating amount of evidence has suggested that there is a contributive role of sympathetic nervous hyperactivity in the pathogenesis of chronic kidney disease (CKD). $\alpha 1-\mathrm{AR}$ promotes an increase in calcium levels in podocytes and adjusts podocyte contraction. Changes in TRPC6 expression and function can directly affect the podocyte cytoskeleton, which is a key component in podocyte injury. This study proposed to clarify the correlation between $\alpha 1-\mathrm{AR}$ activation-induced signal cascade reaction and TRPC6 in human podocytes.
\end{abstract}

Methods: Human podocytes were incubated with the calcium probe Fluo-3/AM. Next, the effects of the $\alpha 1$-AR agonists or antagonists and nonselective TRPC6 blockers on intracellular calcium were observed under laser confocal microscopy. FITC-phalloidin was employed to stain podocytes, and the change of F-actin under the $\alpha 1-\mathrm{AR}$ activation condition was observed.

Results: The $\alpha 1-\mathrm{AR}$ agonist PE (phenylephrine hydrochloride) induced an increase in intracellular $\mathrm{Ca}^{2+}\left(\left[\mathrm{Ca}^{2+}\right] \mathrm{i}\right)$ in human podocytes. Moreover, the downregulation of TRPC6 by siRNA or TRPC blocker could attenuate the PE-induced $\left[\mathrm{Ca}^{2+}\right]$ i elevation in a phospholipase $\mathrm{C}$ (PLC)-dependent pattern. When podocytes were stimulated to the PE, their F-actin fiber cytoskeletal structure was lost. PE subsequently increased the expression of RhoA, and the TRPC6-dependent $\mathrm{Ca}^{2+}$ influx was involved in this process. The abnormal activation of RhoA could result in disturbance of the podocyte skeleton structure, thus leading to podocyte injury.

Conclusions: We concluded that TRPC6 is involved in $\alpha 1-\mathrm{AR}$ activation-induced calcium signal changes in podocytes. Meanwhile, the $\alpha 1-\mathrm{AR}$ agonists can destroy the cell's cytoskeletal structure, which is mediated by TRPC6 via the RhoA/ROCK pathway.

Keywords: TRPC6; $\alpha 1-\mathrm{AR}$; cell cytoskeletal structure; podocytes

Submitted Dec 11, 2019. Accepted for publication Apr 21, 2020.

doi: 10.21037/apm-19-602

View this article at: http://dx.doi.org/10.21037/apm-19-602

\section{Introduction}

Strong evidence has shown that both the kidney and the sympathetic nervous system are vitally involved in the progression and maintenance of hypertension (1). Several studies have demonstrated that sympathetic nerves are involved in controlling the glomerular filtration rate and renin secretion (2). Additionally, sympathetic nerves in the kidney are also involved in the pathogenesis of hypertension, diabetes, and ischemia (3). Nevertheless, the exact effects of sympathetic nervous system activation in podocyte function is unknown. The calcium signal, the most important signaling pathway, participates in the regulation of cell functions after 
the activation of $\alpha 1$-adrenoceptor ( $\alpha 1$-AR) (3). Renal $\alpha 1$ AR activation can increase the calcium levels in podocytes, thereby regulating podocyte function (4). Our team has shown that there is an expression of $\alpha 1-\mathrm{AR}$ in mesangial cells, which can regulate cell proliferation (5). These findings may shed new light on the potential novel therapeutic options for delaying progressive kidney disease. However, at present, $\alpha 1-$ $\mathrm{AR}$ activation-triggered signaling cascades have rarely been illustrated in podocytes.

Transient receptor potential 6 (TRPC6), a prominent member of TRPC channels, is extensively distributed in the glomeruli and renal tubules and is mainly expressed in podocytes (2). As a calcium ion-permeable non-specific cation channel, TRPC6 is involved in multiple intracellular calcium signal changes. A previous study has shown the involvement of TRPC6 upregulation in $\mathrm{Ca}^{2+}$ signaling as well as actin reorganization in podocytes following oxygenglucose deprivation (6). Moreover, gene mutation or abnormal expression of TRPC6 not only leads to genetic focal segmental glomerular sclerosis but also plays a vital role in numerous acquired proteinuric diseases $(7,8)$. $\alpha 1$-AR is a $\mathrm{G}$ protein-coupled receptor, and thus $\alpha 1-\mathrm{AR}$ activation can cause $G$ protein coupling. Their interaction leads to the hydrolysis of phosphatidylinositol-4,5-bisphosphate by phospholipase C (PLC), thereby forming inositol-1, 4,5-trisphosphate (IP3) and diacylglycerol (DAG). Of them, IP3 can activate the cellular store-operated calcium channel to release the intracellular calcium, while DAG can activate the receptor-operated calcium channel to cause persistent inflow of extracellular calcium.

TRPC6 can be activated by PLC-dependent pathway and belongs to the receptor-operated calcium channel, which has been shown to be an important component of intracellular calcium changes following $\alpha 1-\mathrm{AR}$ activation in rabbit venous smooth muscle cells, mouse myocardial cells, nerve cells and vascular smooth muscle cells (9). Therefore, in this study, we aimed to explore the possible role of al-AR activation to stimulate intracellular $\mathrm{Ca}^{2+}\left(\left[\mathrm{Ca}^{2+}\right] \mathrm{i}\right)$ increase by TRPC6 in human podocytes and identify the underlying mechanisms.

Filamentous actin (F-actin) plays a vital role in healthy podocyte foot processes (FPs). By contrast, the disruption of dynamin can trigger the absence of the functional actin network and incongruous membrane signaling, leading to an effacement of FPs and subsequent proteinuria $(10,11)$. RhoA, a member of the Rho protein family, can regulate numerous cell processes, including those involved in the actin cytoskeleton $(12,13)$. TRPC6 and RhoA consist of a molecular complex, and TRPC6-mediated $\mathrm{Ca} 2+$ influx increases RhoA activity (2). RhoA can affect the morphology and function of podocytes. Lipid radicals regulate podocyte homeostatic alterations via redox-sensitive RhoA signaling by causing a shortage of F-actin fibers (14). To this end, we explored whether $\alpha 1$-AR activation could induce TRPC6dependent $\mathrm{Ca}^{2+}$ influx and whether $\mathrm{Ca}^{2+}$ influx could further mediate RhoA activation and as participate in $\alpha 1-\mathrm{AR}$ activation-triggered actin cytoskeleton alterations.

\section{Methods}

\section{Cell culture and transfection}

The human podocyte cell line was kindly offered by Dr. Moin Saleem of Children's Renal Unit and Academic Renal Unit, University of Bristol, Southmead Hospital, Bristol, UK. The cell line was subsequently maintained in line with previous protocols (15). In brief, cells were maintained at $33{ }^{\circ} \mathrm{C}$ in RPMI-1640 medium (HyClone, USA), containing $10 \%$ fetal bovine serum (FBS; HyClone) as well as insulin, transferrin, sodium selenite (ITS; Sigma). Human podocytes between passages 4 and 15 were utilized for experimental assays. Cells were transiently transfected with TRPC6 siRNA (10 $\mu$ M; Santa Cruz, CA, USA) or scrambled siRNA (10 $\mu \mathrm{M}$; Santa Cruz, CA, USA) using X-treme GENE siRNA Transfection Reagent (Roche) in line with the standard protocols. After transfection for 24-48 h, related in vitro assays were performed on the transfected cells.

\section{Fluorescence measurement of intracellular $\mathrm{Ca}^{2+}\left(\left[\mathrm{Ca}^{2+}\right]\right.$ i)}

$\left[\mathrm{Ca}^{2+}\right]$ i levels were established according to previous protocols (16). Briefly, human podocytes were incubated with $1 \%$ normal saline supplemented with Pluronic F-127 $(0.03 \%$, Sigma) as well as Fluo-3/AM ( $3 \mu \mathrm{M}$, Molecular Probes) at $37{ }^{\circ} \mathrm{C}$ for $45 \mathrm{~min}$, followed by stimulation with diverse inhibitors agonists or antagonists as indicated in the result section, including U73122 (a PLC inhibitor, Sigma), and nonselective TRPC6 blockers including 2-APB (Sigma) and SKF-96365 (Sigma). Afterward, a laser confocal scanning microscope (FV300; Olympus, Japan) was used to determine the fluorescence intensity of Fluo-3 in podocytes. Additionally, the actual fluorescence intensity was divided by the average baseline fluorescence intensity to display the pseudo-ratio value of $\left[\mathrm{Ca}^{2+}\right]$ i. Results from 20 to 40 cells were summarized in a single run, and this assay was performed in triplicate. 


\section{Western blot (WB)}

WB was conducted in line with standard protocols. In brief, the membranes reacted with appropriate primary antibodies, including polyclonal rabbit anti-TRPC6 (1:200, Alomone Labs, Israel), anti-RhoA (1:500, Santa Cruz, CA, USA) and anti-glyceraldehyde-3-phosphate dehydrogenase (GAPDH) antibody (1:1,000, ZSGB-Bio). The Odyssey infrared imaging system (LI-COR, Lincoln) was used for visualization and quantification of the bands.

\section{Fluorescence staining of F-actin}

For immunofluorescence (IF) staining, podocytes were first cultured on coverslips and further stained according to the manufacturers' instructions. Cells were fixed with $4 \%$ paraformaldehyde (PFA) for $15 \mathrm{~min}$, permeabilized with $0.4 \%$ Triton X-100, then blocked with $1 \%$ bovine serum albumin (BSA) in phosphate-buffered saline (PBS), followed by staining with phalloidin-FITC (5 $\mu \mathrm{g} / \mathrm{mL}$, Sigma). Afterward, a laser scanning confocal microscope (Olympus, Japan) was used to observe and photograph representative images.

\section{Rbo A activation assay}

The Rho activation assay kit (Cytoskeleton, Inc.) was purchased to observe the RhoA activity according to the manufacturer's instructions. After cell lysis and centrifugation, the supernatant was collected for incubation with RhotekinRho binding domain (RBD) glutathione affinity beads, which specifically bound to GTP-bound RhoA. WB was then employed to measure the protein expression of total RhoA after normalization to GTP-bound RhoA densitometric units. All results were repeated 3 times.

\section{Statistical analysis}

All the data are displayed as means \pm standard error (SEM). Two-tailed Student's $t$-test or one-way analysis of variance (ANOVA) was utilized for comparison between groups, along with Dunnett's test. A P value $<0.05$ suggested statistical significance.

\section{Results}

a1-AR agonists stimulate an elevation in $\left[\mathrm{Ca}^{2+}\right]$ i in buman podocytes

To explore the possible relationship between the protein expression of $\alpha 1-\mathrm{AR}$ and functional receptors presence, $\alpha 1$-AR specific agonist phenylephrine hydrochloride (PE, $10 \mu \mathrm{M})$ was employed to stimulate Fluo-3/AM-loaded human podocytes. The calcium ion in podocytes was rapidly increased and reached a peak under PE stimulation at $100 \mathrm{~s}$, remained stable continuously, and finally returned to the baseline level (Figure 1A). Pretreatment of podocytes with $200 \mathrm{nM}$ prazosin, a selective $\alpha 1-\mathrm{AR}$ antagonist, could lead to significant suppression of PE-induced $\left[\mathrm{Ca}^{2+}\right] \mathrm{i}$ elevation (Figure 1B). To further investigate the potential role of $\mathrm{Ca}^{2+}$ influx in $\mathrm{PE}$-induced $\left[\mathrm{Ca}^{2+}\right]$ i elevation, $\mathrm{PE}$ was used to stimulate podocytes without extracellular $\mathrm{Ca}^{2+}$. Consequently, the application of PE stimulation in podocytes still led to the transient increase of $\left[\mathrm{Ca}^{2+}\right] \mathrm{i}$ in podocytes (Figure 1C). However, the peak of PE-induced $\left[\mathrm{Ca}^{2+}\right]$ i elevation was slight without calcium induction, almost without a steady plateau, suggesting that an extracellular $\mathrm{Ca}^{2+}$ entry was very likely to participate in $\left[\mathrm{Ca}^{2+}\right]$ $i$ elevation by $\alpha 1-A R$ activation in human podocytes.

\section{The role of the PLC signaling pathway in a1-AR activation-induced $\left[\mathrm{Ca}^{2+}\right]$ i increase in podocytes}

To further determine whether the PLC-dependent pathway participated in $\alpha 1-\mathrm{AR}$ activation-induced $\left[\mathrm{Ca}^{2+}\right]$ i elevation, $\alpha 1-\mathrm{AR}$ agonists were utilized to stimulate the PLC inhibitor U73122 $(10 \mu \mathrm{M})$. As shown in Figure $1 D$, the administration of U73122 almost completely abolished the PE-induced $\left[\mathrm{Ca}^{2+}\right]$ i elevation, confirming the PLC-dependent pattern in PE-induced $\left[\mathrm{Ca}^{2+}\right]$ i elevation in podocytes.

\section{Activation of a1-AR induces TRPC6-dependent $\mathrm{Ca}^{2+}$ influx}

Nonselective TRPC channel blockers, SKF96365 $(30 \mu \mathrm{M})$ and 2-APB $(100 \mu \mathrm{M})$ were employed to pharmacologically abolish TRPC, which further significantly suppressed the PE-induced $\left[\mathrm{Ca}^{2+}\right]$ i increase (Figure $2 A, B, C, D$ ). The above outcomes collectively indicate that TRPC-dependent $\mathrm{Ca}^{2+}$ entry participated in the $\mathrm{PE}$-induced $\left[\mathrm{Ca}^{2+}\right]$ i elevation $(\mathrm{P}<0.001 ; \mathrm{n}=3)$. In order to determine whether TRPC6 contributed to the $\alpha 1-\mathrm{AR}$ activation-induced $\left[\mathrm{Ca}^{2+}\right] \mathrm{i}$ elevation, siRNA targeting TRPC6 was transfected into human podocytes to downregulate TRPC6 expression, followed by analysis to validate the knockdown efficiency of TRPC6-siRNA (data not shown). As displayed in Figure 2E,F, PE-induced $\left[\mathrm{Ca}^{2+}\right]$ i elevation was significantly suppressed by transfection with TRPC6 siRNA $(\mathrm{P}<0.001$; 

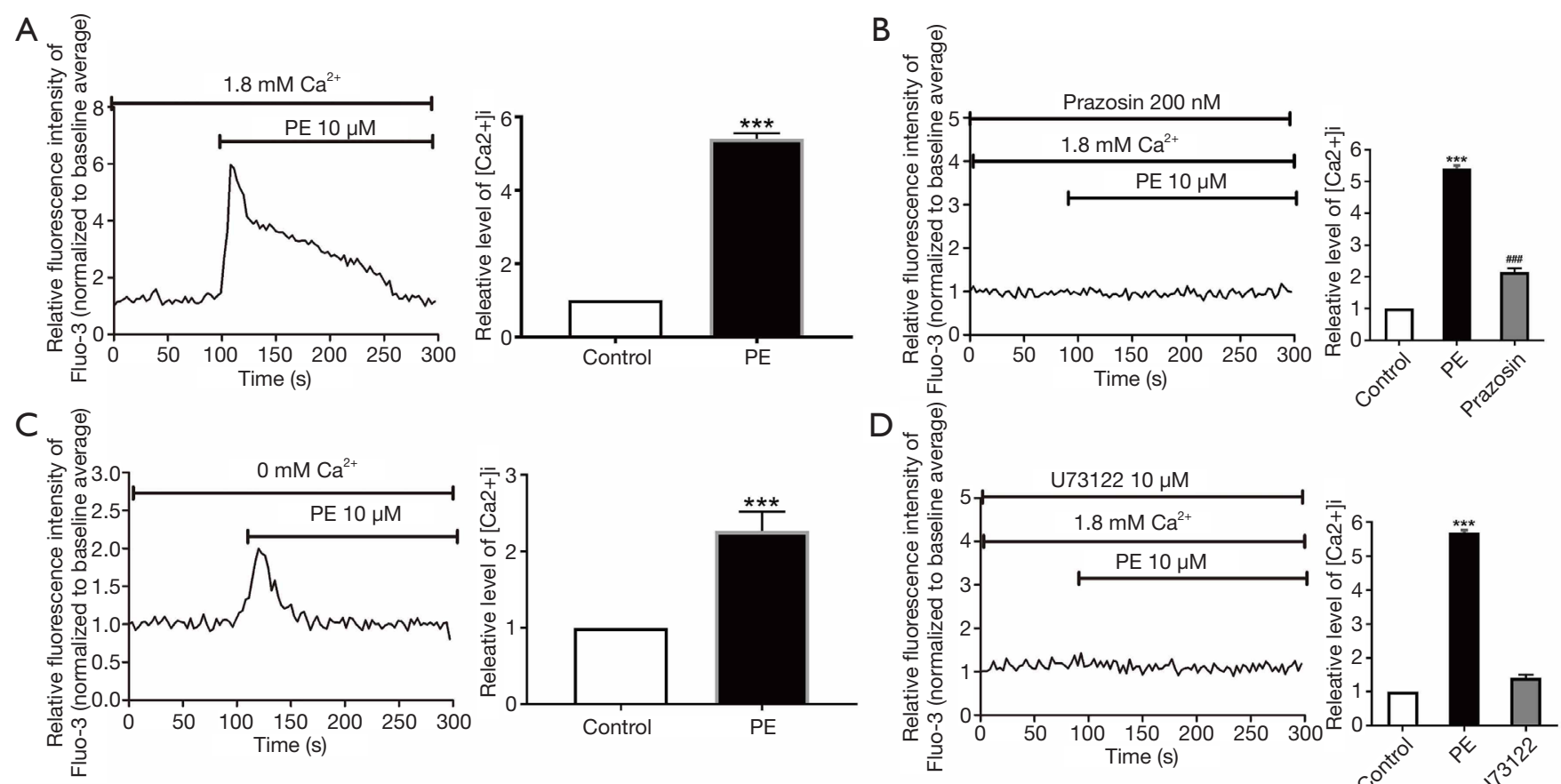

Figure $1 \alpha 1$-AR agonist-induced the increased $\left[\mathrm{Ca}^{2+}\right] \mathrm{i}$ in human podocytes. Confocal microscopy using Fura-3 fluorescent dye was used to observe changes in the $\mathrm{Ca}^{2+}$ concentration. (A) A representative trace showing that $\alpha 1-\mathrm{AR}$ agonist $\mathrm{PE}$ induced a [Ca $\left.{ }^{2+}\right] \mathrm{i}$ increase in the

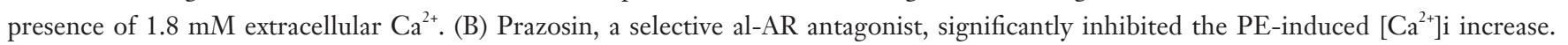
(C) The PE-induced $\left[\mathrm{Ca}^{2+}\right] \mathrm{i}$ increase peak was small in the absence of calcium induction, which almost had no steady plateau. (D) The PLC inhibitor U73122 could almost completely suppress the PE-induced $\left[\mathrm{Ca}^{2+}\right] \mathrm{i}$ increase. The results were from at least 3 independent experiments; 20-30 cells were selected in each trial. ***, $\mathrm{P}<0.001$ vs. Ctrl $\mathrm{n}=3 ;{ }^{\# \# \#,} \mathrm{P}<0.001$ vs. $\mathrm{PE}$.

$\mathrm{n}=3$ ), in comparison with transfection with scrambled small interfering RNA (siRNA). The above outcomes demonstrated that TRPC6 was involved in the $\alpha 1$-AR activation-induced increase of $\left[\mathrm{Ca}^{2+}\right]$ i.

\section{a1-AR activation increases the protein expression of TRPC6 in podocytes}

At present, the impacts of $\alpha 1-\mathrm{AR}$ activation on inducing the protein expression of TRPC6 in podocytes remains largely undefined. Herein, WB was employed to examine the changes in TRPC6 protein expression in human podocytes following the administration of $\alpha 1-\mathrm{AR}$ specific agonist $\mathrm{PE}$. As shown in Figure $3 \mathrm{~A}$, the administration of $10 \mu \mathrm{M}$ PE for $48 \mathrm{~h}$ could elevate the protein expression of TRPC6 in podocytes.

\section{a1-AR activation induces the TRPC6-dependent $\mathrm{Ca}^{2+}$ inflow to increase the RboA activation}

Affinity pull-down assay through the GST fusion protein rhotekin was used to directly examine the RhoA activity, and was able to recognize only the active form of RhoA (GTP-RhoA). As displayed in Figure 3B, the administration of $\alpha 1-\mathrm{AR}$ agonist $\mathrm{PE}$ for $48 \mathrm{~h}$ significantly increased RhoA activity compared with the normal group. This was likely be due to PE-induced TRPC6 activation, as PE-induced elevation of RhoA activity following transfection with TRPC6 siRNA was attenuated compared with the normal group, non-transfection group, and scramble siRNA group $(\mathrm{P}<0.05 ; \mathrm{n}=3)$. The above outcomes suggested that TRPC6 plays a role in the $\alpha 1-\mathrm{AR}$ activation-induced $\mathrm{Ca}^{2+}$ inflow, further contributing to RhoA activation.

\section{TRPC6 destroys the cytoskeleton stress fiber via the Rho $A$ pathway in response to $\alpha 1-A R$ activation}

To investigate the functional effects of TRPC 6 on $\alpha 1-\mathrm{AR}$ activation-induced skeleton stress fiber, FITC-phalloidin was employed to stain podocytes. As shown in Figure 4A, well-developed $\mathrm{F}$-actin fibers were detected in normal 

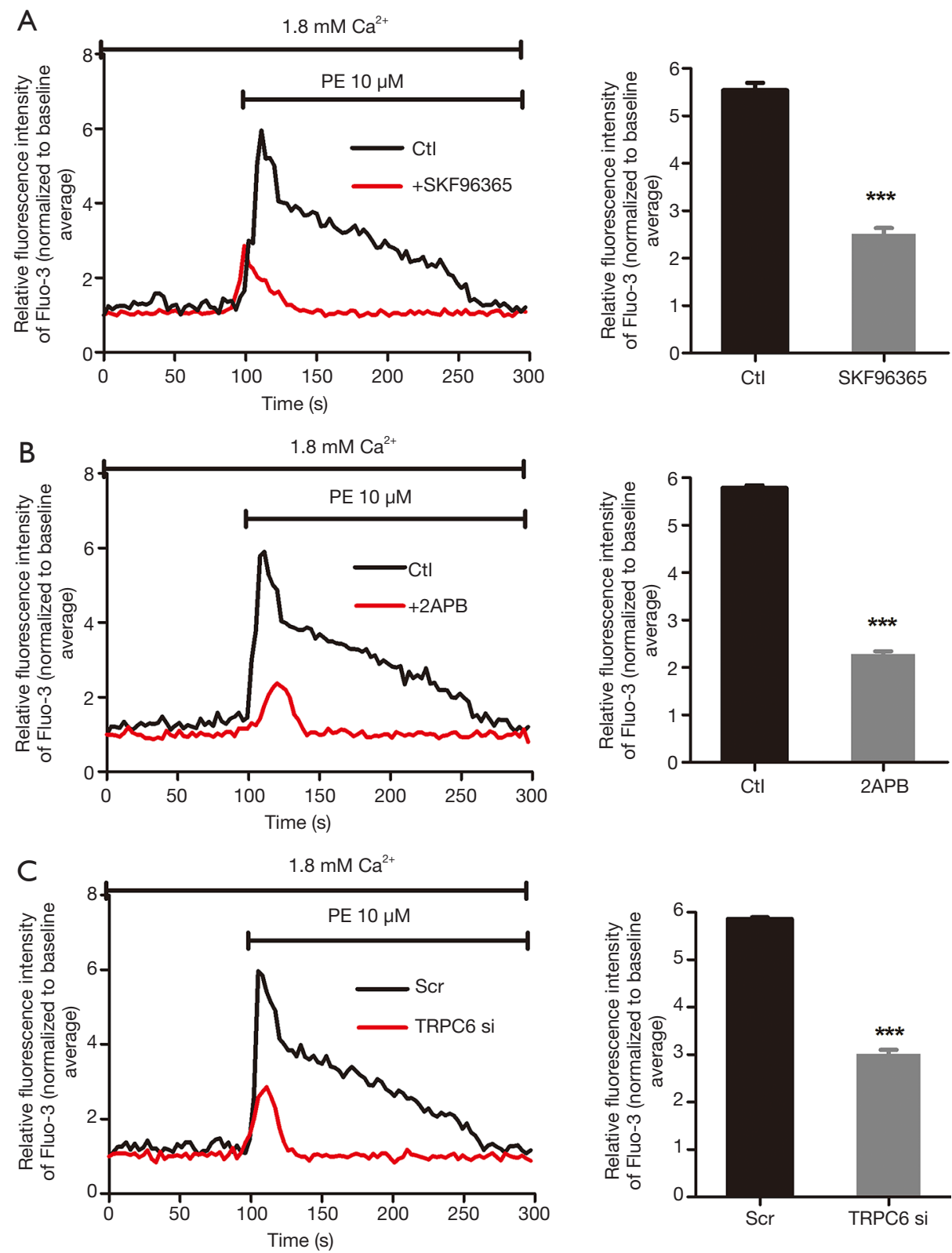

Figure 2 Activation of $\alpha 1-\mathrm{AR}$ induces TRPC6-dependent $\mathrm{Ca}^{2+}$ influx. Confocal microscopy using Fura-3 fluorescent dye was used to observe changes in the $\mathrm{Ca}^{2+}$ concentration. (A and B) SKF96365 $(30 \mu \mathrm{M})$ significantly inhibited the $\left[\mathrm{Ca}^{2+}\right]$ i expression induced by PE (***, $\mathrm{P}<0.001$ vs. Ctrl $\mathrm{n}=3)$. (C and D) 2-APB $(100 \mu \mathrm{M})$ significantly inhibited the expression of $\left[\mathrm{Ca}^{2+}\right] \mathrm{i}$ induced by $\mathrm{PE}(* * *, \mathrm{P}<0.001$ vs. Ctrl $\mathrm{n}=3)$. (E and F) TRPC6 siRNA could remarkably inhibit the expression of $\left[\mathrm{Ca}^{2+}\right]$ i increased by $\mathrm{PE}(\mathrm{P}<0.001 ; \mathrm{n}=3) .\left({ }^{* * *}, \mathrm{P}<0.001 v s\right.$. Scr $\left.\mathrm{n}=3\right)$. The number of trials $=3 ; 20-30$ cells were selected in each trial.

podocytes. F-actin manifested as the yellow-green, long, and fine filament running along the whole-length of podocytes after FITC-phalloidin staining. The treatment with the culture medium containing $10 \mu \mathrm{M} \alpha 1$-AR agonist $\mathrm{PE}$ caused a rearrangement of $\mathrm{F}$-actin, rupture of stress fibers, and reduction of the number of orderly arranged fibers, in comparison with those in the normal group. As expected, after transfection with TRPC6-siRNA and the administration of TRPC channel inhibitor SKF-96365, the stress fibers partially ruptured, the number of orderly 
A

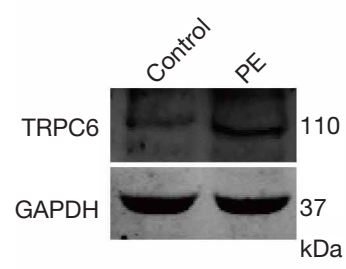

B

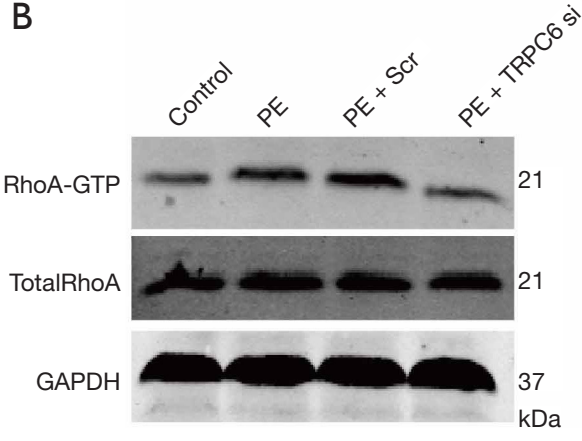

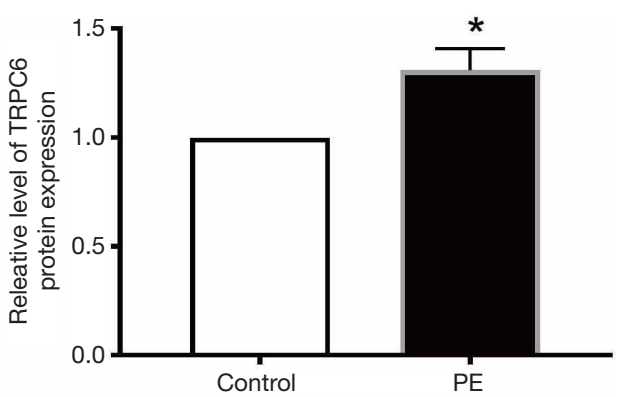

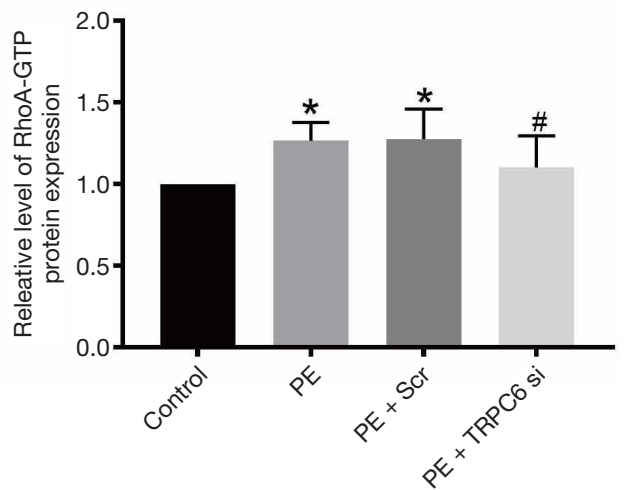

Figure $3 \alpha 1$-AR activation induced the TRPC6-dependent $\mathrm{Ca}^{2+}$ inflow to increase the RhoA activation. (A) Compared with normal podocytes, the application of $10 \mu \mathrm{M} \mathrm{PE}$ to treat podocytes for $48 \mathrm{~h}$ could upregulate the TRPC6 protein expression $\left({ }^{*}, \mathrm{P}<0.05\right.$ vs. Ctrl, $\mathrm{n}=3$ ). (B) Compared with the normal group, RhoA activity was markedly enhanced after $48 \mathrm{~h}$ of treatment with $\alpha 1-\mathrm{AR}$ agonist PE; relative to the normal group, the non-transfection group and scramble siRNA group attenuated the PE-induced increase in RhoA activity ${ }^{*}$, $\mathrm{P}<0.05$ vs. Ctrl;,, $\mathrm{P}<0.05$ vs. PE + Scr, n=3).

arranged fibers slightly increased, and the PE-induced F-actin rearrangement slightly recovered, compared with those in the PE stimulation group (Figure 4A,B). To determine whether the RhoA pathway was the downstream pathway of the involvement of TRPC6 in the PE-induced podocyte skeleton destruction, the RhoA/ROCK inhibitor Y-27632 was used for intervention. Consequently, the number of ruptured stress fibers decreased, and the number of orderly arranged fibers slightly increased, in comparison with those in the PE stimulation group (Figure 4C). The above results indicate the relationship between TRPC6mediated RhoA pathway and $\alpha 1-\mathrm{AR}$ activation-induced alterations in the cytoskeleton.

\section{Discussion}

The kidneys, which are rich in afferent and efferent sympathetic nerves, play crucial physiological roles in the modulation of renal functions. Evidence from several studies supports the speculation autosomal dominant polycystic kidney disease manifests as sympathetic renal hyperactivity (17). Moreover, recent studies have shown that renal sympathetic inhibition or denervation is likely to partially reduce or even prohibit renal destruction triggered by ischemia/reperfusion injury (18). In addition, the importance of renal nerves has been recently discussed in relation to sympathetic activity in other vascular beds, arterial pressure, and long-term hypertension (19). However, the possible roles of renal sympathetic nerves in podocytes have not been extensively investigated. To this end we here demonstrate that $\alpha 1-\mathrm{AR}$ agonists can trigger $\left[\mathrm{Ca}^{2+}\right]$ i elevation in human podocytes.

$\alpha 1-\mathrm{AR}$ participates in diverse physiological functions controlled by endogenous catecholamines, noradrenaline, and adrenaline, which is also a useful therapeutic target (20). Functionally, $\alpha 1-\mathrm{AR}$ utilizes intracellular $\mathrm{Ca}^{2+}$ as a second messenger for the regulation of cellular function in multiple types of cells. For example, smooth muscle contractions to exogenous agonists are particularly mediated by $\alpha 1-$ AR (7). Nakladal et al. suggested that (R)-enantiomer of 
A

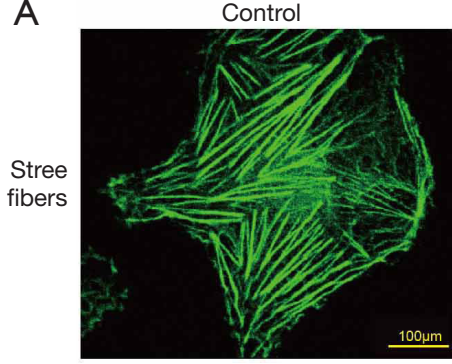

B

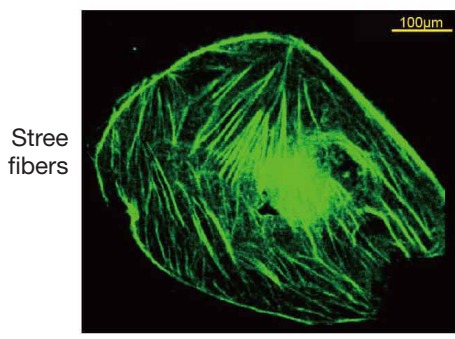

C

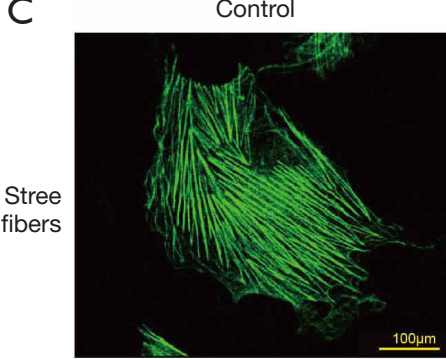

PE

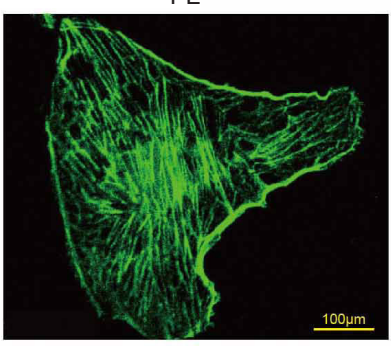

TRPC6 si

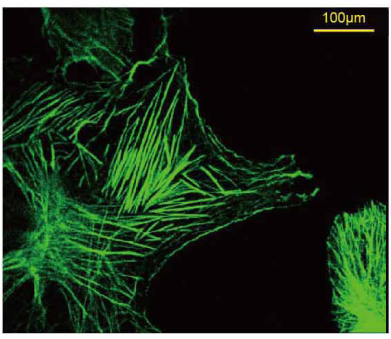

PE

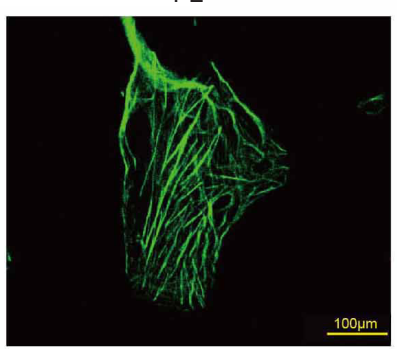

SKF96365
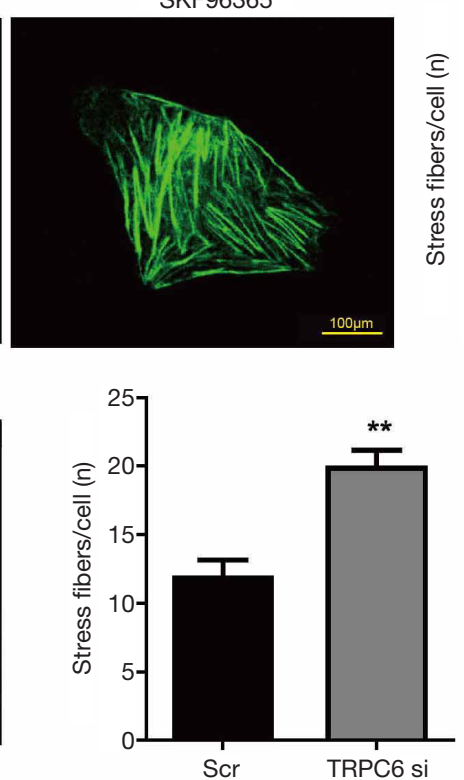

Y-27632

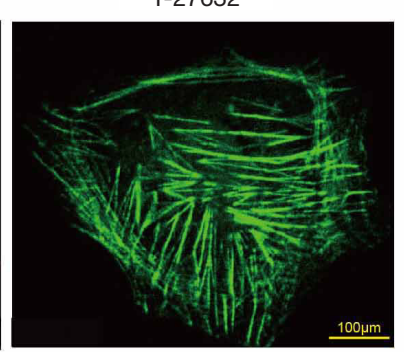

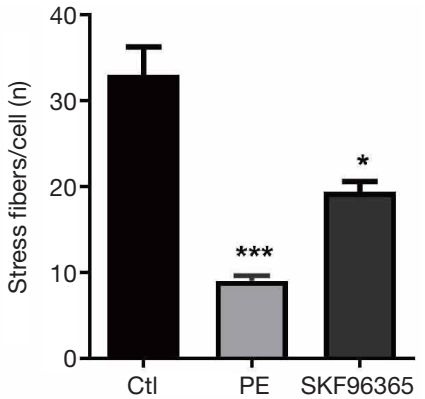

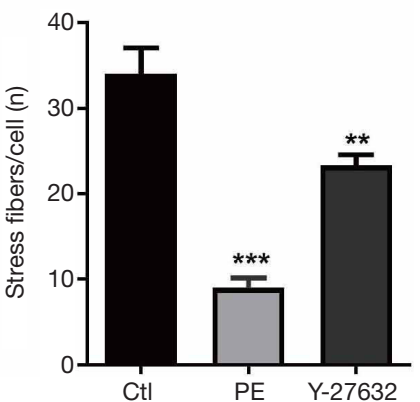

Figure 4 a1-AR activation induced TRPC6 to destroy the cytoskeleton stress fiber through the RhoA pathway. FITC-labeled phalloid was employed to evaluate the changes of the podocyte skeleton by stress fiber. (A) SKF-96365 could rupture the stress fibers induced by PE (***, $\mathrm{P}<0.001$ vs. Ctrl; ${ }^{*}, \mathrm{P}<0.05$ vs. $\left.\mathrm{PE}, \mathrm{n}=3\right)$. (B) TRPC6-siRNA ruptured the stress fibers compared with the scramble siRNA group ${ }^{* *}, \mathrm{P}<0.01$ vs. Scr, $\mathrm{n}=3)$. (C) $\mathrm{Y}-27632$ increased the stress fiber number induced by the $\mathrm{PE}$ group ${ }^{* * *}, \mathrm{P}<0.001$ vs. Ctrl; ${ }^{* *}, \mathrm{P}<0.01$ vs. $\left.\mathrm{PE}, \mathrm{n}=3\right)$.

the 6-chromanol derivate SUL-121 improves renal graft perfusion via antagonism of the $\alpha 1-\mathrm{AR}(4)$. Cabrita et al. showed that $\mathrm{Ca}^{2+}$ signaling is important in TMEM16A driving renal cyst growth (21). Our experimental findings reveal that activation of al-AR could mediate $\mathrm{Ca}^{2+}$ influx through TRPC6 in human podocytes. Consistent with research on other cell types (22), we also found that the PE-induced increase in intracellular calcium was derived from the release of intracellular calcium and inflow of extracellular calcium.

The physiological function in podocytes vitally relies on appropriate intracellular calcium handling, while excessive calcium influx in podocytes might cause FP effacement, apoptosis and subsequent glomeruli injury.
TRPC6 is considered a critical protein that is responsible for calcium flux in podocytes, while hyper-activation or mutation of TRPC6 channel activity can result in glomeruli injury progression (23). Moreover, TRPC6 is related to the pathogenesis of familial forms of focal segmental glomerulosclerosis (FSGS) (7), while $\alpha 1$-AR belongs to the $\mathrm{G}$ protein-coupled receptor, whose activation can produce IP3 and DAG. TRPC6 is widely acknowledged to function as a DAG-activated channel. Therefore, it is reasonable to speculate that the PLC-dependent generation of DAG could trigger TRPC6 following a1-AR stimulation. Herein, we have shown that the PLC inhibitor almost entirely suppressed the $\alpha 1-\mathrm{AR}$ activation-induced $\left[\mathrm{Ca}^{2+}\right]$ $\mathrm{i}$ elevation in podocytes, which is consistent with previous 
outcomes observed in myocardial cells (24). Moreover, we demonstrate that $\alpha 1-\mathrm{AR}$ agonist $\mathrm{PE}$ stimulation triggers not only $\mathrm{Ca}^{2+}$ release but also TRPC6-dependent $\mathrm{Ca}^{2+}$ entry in human podocytes. Considering the glomerular sclerosis induced by changes in the TRPC6, we preliminarily posit that $\alpha 1$-AR activation-induced changes in TRPC6 activity is vitally involved in podocyte injury and glomerular sclerosis development.

The specialized morphology of podocytes, visceral epithelial cells of the renal glomerulus, form the selective glomerular filtration barrier. The cytoskeleton of podocytes consists of intermediate filaments, microtubules, and F-actin. Loss of over $30 \%$ of podocytes is harmful to the glomerulus, subsequently causing glomerulosclerosis and chronic kidney disease (CKD), a common complication of diabetes. Thus, it is of great clinical significance to preserve podocytes (25). Cytoskeletal structure plays an essential role in maintaining appropriate $\mathrm{FP}$ and the filtration barrier integrity (26). According to our present findings, the exposure of podocytes to a1-AR agonist PE could result in a loss of cytoskeletal structure of F-actin fiber. After knockdown of TRPC6 expression by transfecting TRPC6siRNA, the $\alpha 1$-AR agonist PE could attenuate the podocyte cytoskeletal structure destruction. Additionally, the administration of $\alpha 1-\mathrm{AR}$ agonist $\mathrm{PE}$ in podocytes could also induce the activation of RhoA, which was notably attenuated by TRPC6 siRNA. The abnormal activation of RhoA could result in disturbance of the cytoskeletal structure and further suppress the formation in podocytes (27). However, previous studies have demonstrated that excessive or scarce RhoA activity is equally harmful to podocytes by increasing or decreasing RhoA activity to subsequently cause proteinuric kidney disease $(28,29)$. In our experiments, hyperactive RhoA resulted in a cortical rearrangement of F-actin fibers. When the RhoA/ROCK inhibitor Y27632 was used, the $\alpha 1-\mathrm{AR}$ agonist PE could decrease the destruction of the podocyte skeleton. On the other hand, we can speculate that a hyperactive RhoA is destructive and can cause cytoskeletal rearrangement and $\mathrm{F}$-actin fiber loss. Consistent with this, our present outcomes also show the loss of F-actin fibers and RhoA activation in a similar fashion. If the actin cytoskeleton were repaired, the early injury of podocytes might be irreversible, while persistent and chronic podocyte injury would result in the loss of glomerular function. However, the precise mechanism for TRPC6 over-load a1-AR activation-induced calcium signal changes in podocytes is still unclear, and is of great significance to our future research.

\section{Conclusions}

The activation of $\alpha 1$-AR can upregulate TRPC6 expression and increase calcium inflow in podocytes. Meanwhile, TRPC6 can induce RhoA activation, which participates in the $\alpha 1$-AR agonist PE-induced podocyte injury through the RhoA mechanism. Moreover, these outcomes offer a new pathway that could be the first step for a better understanding of how $\alpha 1-\mathrm{AR}$ activation affects podocytes, which is of great clinical significance in CKD.

\section{Acknowledgments}

Funding: This work was supported by the National Nature Science Foundation of China (81441023).

\section{Footnote}

Data Sharing Statement: Available at http://dx.doi. org/10.21037/apm-19-602

Conflicts of Interest: All authors have completed the ICMJE uniform disclosure form (available at http://dx.doi. org/10.21037/apm-19-602). The authors have no conflicts of interest to declare.

Ethical Statement: The authors are accountable for all aspects of the work in ensuring that questions related to the accuracy or integrity of any part of the work are appropriately investigated and resolved.

Open Access Statement: This is an Open Access article distributed in accordance with the Creative Commons Attribution-NonCommercial-NoDerivs 4.0 International License (CC BY-NC-ND 4.0), which permits the noncommercial replication and distribution of the article with the strict proviso that no changes or edits are made and the original work is properly cited (including links to both the formal publication through the relevant DOI and the license). See: https://creativecommons.org/licenses/by-nc-nd/4.0/.

\section{References}

1. Siedlinski M, Jozefczuk E, Xu X, et al. White Blood Cells and Blood Pressure: A Mendelian Randomization Study. 
Circulation 2020;141:1307-17.

2. Yu J, Zhu C, Yin J, et al. Tetrandrine Suppresses Transient Receptor Potential Cation Channel Protein 6 Overexpression- Induced Podocyte Damage via Blockage of RhoA/ROCK1 Signaling. Drug Des Devel Ther 2020;14:361-70.

3. Lambert E, Schlaich $M$. The role of renal sympathetic nerves in ischemia reperfusion injury. Auton Neurosci 2017;204:105-11.

4. Nakladal D, Buikema H, Romero AR, et al. The (R)enantiomer of the 6-chromanol derivate SUL-121 improves renal graft perfusion via antagonism of the alpha1-adrenoceptor. Sci Rep 2019;9:13.

5. Kong F, Ma L, Zou L, et al. Alpha1-Adrenergic Receptor Activation Stimulates Calcium Entry and Proliferation via TRPC6 Channels in Cultured Human Mesangial Cells. Cell Physiol Biochem 2015;36:1928-38.

6. Lopez JR, Uryash A, Faury G, et al. Contribution of TRPC Channels to Intracellular $\mathrm{Ca}(2+)$ Dyshomeostasis in Smooth Muscle From mdx Mice. Front Physiol 2020;11:126.

7. Wang Q, Tian X, Wang Y, et al. Role of Transient Receptor Potential Canonical Channel 6 (TRPC6) in Diabetic Kidney Disease by Regulating Podocyte Actin Cytoskeleton Rearrangement. J Diabetes Res 2020;2020:6897390.

8. Hall G, Wang L, Spurney RF. TRPC Channels in Proteinuric Kidney Diseases. Cells 2019;9:44.

9. DeLalio LJ, Billaud M, Ruddiman CA, et al. Constitutive SRC-mediated phosphorylation of pannexin 1 at tyrosine 198 occurs at the plasma membrane. J Biol Chem 2019;294:6940-56.

10. Liu J, Huang S, Li F, et al. Sympathetic Nerves Positively Regulate Eosinophil-Driven Allergic Conjunctivitis via alpha1-Adrenergic Receptor Signaling. Am J Pathol 2020;190:1298-308.

11. Alcantara-Hernandez R, Carmona-Rosas G, HernandezEspinosa DA, et al. Glycogen Synthase Kinase-3 modulates alpha1A-adrenergic receptor action and regulation. Eur J Cell Biol 2020;99:151072.

12. Kloc M, Uosef A, Wosik J, et al. RhoA Pathway and Actin Regulation of the Golgi/Centriole Complex. Results Probl Cell Differ 2019;67:81-93.

13. Xia M, Chen Y, He Y, et al. Activation of the RhoA-YAPbeta-catenin Signaling Axis Promotes the Expansion of Inner Ear Progenitor Cells in 3D Culture. Stem Cells 2020. [Epub ahead of print].

14. Cao J, Yang T, Tang D, et al. Increased expression of
GEF-H1 promotes colon cancer progression by RhoA signaling. Pathol Res Pract 2019;215:1012-9.

15. Yang H, Zhao B, Liao C, et al. High glucose-induced apoptosis in cultured podocytes involves TRPC6dependent calcium entry via the RhoA/ROCK pathway. Biochem Biophys Res Commun 2013;434:394-400.

16. Liao C, Yang H, Zhang R, et al. The upregulation of TRPC6 contributes to $\mathrm{Ca}(2)(+)$ signaling and actin assembly in human mesangial cells after chronic hypoxia. Biochem Biophys Res Commun 2012;421:750-6.

17. Rovella V, Scimeca M, Giannini E, et al. Morphological evaluation of sympathetic renal innervation in patients with autosomal dominant polycystic kidney disease. J Nephrol 2020;33:83-9.

18. Guvenc M, Cellat M, Uyar A, et al. Nobiletin Protects from Renal Ischemia-Reperfusion Injury in Rats by Suppressing Inflammatory Cytokines and Regulating iNOS-eNOS Expressions. Inflammation 2020;43:336-46.

19. Osborn JW, Foss JD. Renal Nerves and Long-Term Control of Arterial Pressure. Compr Physiol 2017;7:263-320.

20. Akinaga J, Garcia-Sainz JA, A SP. Updates in the function and regulation of alpha1 -adrenoceptors. Br J Pharmacol 2019;176:2343-57.

21. Cabrita I, Buchholz B, Schreiber R, et al. TMEM16A drives renal cyst growth by augmenting $\mathrm{Ca}(2+)$ signaling in M1 cells. J Mol Med (Berl) 2020;98:659-71.

22. Machuki JO, Zhang HY, Geng J, et al. Estrogen regulation of cardiac cAMP-L-type $\mathrm{Ca}(2+)$ channel pathway modulates sex differences in basal contraction and responses to beta2AR-mediated stress in left ventricular apical myocytes. Cell Commun Signal 2019;17:34.

23. Staruschenko A, Spires D, Palygin O. Role of TRPC6 in Progression of Diabetic Kidney Disease. Curr Hypertens Rep 2019;21:48.

24. Mohl MC, Iismaa SE, Xiao XH, et al. Regulation of murine cardiac contractility by activation of alpha(1A)adrenergic receptor-operated $\mathrm{Ca}(2+)$ entry. Cardiovasc Res 2011;91:310-9.

25. Szrejder M, Piwkowska A. AMPK signalling: Implications for podocyte biology in diabetic nephropathy. Biol Cell 2019;111:109-20.

26. Iwata $W$, Unoki-Kubota $H$, Kato H, et al. Podocytespecific deletion of tubular sclerosis complex 2 promotes focal segmental glomerulosclerosis and progressive renal failure. PLoS One 2020;15:e0229397.

27. Breuzard G, Pagano A, Bastonero S, et al. Tau regulates the microtubule-dependent migration of glioblastoma cells via the Rho-ROCK signaling pathway. J Cell Sci 
2019;132:222851.

28. Matsuda J, Maier M, Aoudjit L, et al. ARHGEF7 (betaPIX) Is Required for the Maintenance of Podocyte Architecture and Glomerular Function. J Am Soc Nephrol 2020;31:996-1008.

Cite this article as: Wang D, Wang Q, Ji T, Yang H, Kong F, Jiao J. The role of TRPC6 in a1-AR activation-induced calcium signal changes in human podocytes. Ann Palliat Med 2020;9(4):1596-1605. doi: 10.21037/apm-19-602
29. Johnson AC, Wu W, Attipoe EM, et al. Loss of Arhgef11 in the Dahl Salt-Sensitive Rat Protects Against Hypertension-Induced Renal Injury. Hypertension 2020;75:1012-24. 